\title{
DDX3X and DDX3Y are redundant in protein synthesis
}

\author{
SRIVATS VENKATARAMANAN, ${ }^{1,3}$ MARGARET GADEK, ${ }^{1}$ LORENZO CALVIELLO, ${ }^{1,4}$ KEVIN WILKINS, ${ }^{1}$ \\ and STEPHEN N. FLOOR ${ }^{1,2}$ \\ ${ }^{1}$ Department of Cell and Tissue Biology, University of California, San Francisco, San Francisco, California 94143, USA \\ ${ }^{2}$ Helen Diller Family Comprehensive Cancer Center, University of California, San Francisco, San Francisco, California 94143, USA
}

\begin{abstract}
DDX3 is a DEAD-box RNA helicase that regulates translation and is encoded by the X-and Y-linked paralogs DDX3X and DDX3Y. While DDX3X is ubiquitously expressed in human tissues and essential for viability, DDX3Y is male-specific and shows lower and more variable expression than DDX3X in somatic tissues. Heterozygous genetic lesions in DDX3X mediate a class of developmental disorders called DDX3X syndrome, while loss of DDX3Y is implicated in male infertility. One possible explanation for female-bias in DDX3X syndrome is that DDX3Y encodes a polypeptide with different biochemical activity. In this study, we use ribosome profiling and in vitro translation to demonstrate that the $X$ - and $Y$-linked paralogs of DDX3 play functionally redundant roles in translation. We find that transcripts that are sensitive to DDX3X depletion or mutation are rescued by complementation with DDX3Y. Our data indicate that DDX3X and DDX3Y proteins can functionally complement each other in the context of mRNA translation in human cells. DDX3Y is not expressed in a large fraction of the central nervous system. These findings suggest that expression differences, not differences in paralog-dependent protein synthesis, underlie the sex-bias of DDX3X-associated diseases.
\end{abstract}

Keywords: translational control; DEAD-box proteins; RNA; sex differences

\section{INTRODUCTION}

DDX3X is a ubiquitously expressed ATPase and RNA-helicase encoded by an essential gene on the X-chromosome, DDX3X. DDX3X escapes $X$-inactivation in a wide range of tissues (Cotton et al. 2015). Mutations in DDX3X are associated with numerous pathologies, including cancers like medulloblastoma (Kool et al. 2014; Floor et al. 2016; Oh et al. 2016), chronic lymphocytic leukemia (Ojha et al. 2015), squamous cell carcinoma (Stransky et al. 2011), Burkitt's lymphoma (Gong et al. 2021), and many others (Sharma and Jankowsky 2014). Heterozygous missense or loss-of-function mutations in DDX3X are also implicated in intellectual disability and autism-spectrum disorders in females (lossifov et al. 2014; Snijders Blok et al. 2015; Yuen et al. 2017; Takata et al. 2018; Wang et al. 2018; Ruzzo et al. 2019; Scala et al. 2019; Lennox et al. 2020), with the severity of phenotype correlating with the degree of reduction in DDX3X catalytic activity (Lennox et al. 2020).

DDX3X has a Y-chromosome paralog, DDX3Y, and the protein products of these two genes are more than $90 \%$ identical

\footnotetext{
3Present address: Sardona Therapeutics, San Francisco, CA 94107, USA

${ }^{4}$ Present addresses: Functional Genomics Centre, Human Technopole, Milan 20157, Italy; Computational Biology Centre, Human Technopole, Milan 20157, Italy

Corresponding author: stephen.floor@ucsf.edu

Article is online at http://www.rnajournal.org/cgi/doi/10.1261/rna. 078926.121 . Freely available online through the RNA Open Access option.
}

(Ditton et al. 2004). DDX3Y is located within the Azoospermia Factor a (AZFa) locus nested within the nonrecombining region of the $Y$ chromosome (Kotov et al. 2017). Expression of DDX3Y alone has been shown to rescue the specific infertility phenotype caused by deletion of AZFa (Ramathal et al. 2015), although these findings have been disputed in mice (Matsumura et al. 2019). DDX3Y mRNA is broadly expressed across tissues, but activation of a testis-specific distal promoter produces a transcript isoform with a distinct $5^{\prime}$ leader that also contributes to its translational control (Jaroszynski et al. 2011). Because of this, expression of DDX3Y was thought to be testis-specific, but later work suggested that DDX3Y is expressed more broadly in tissues across the human body (Uhlen et al. 2015) (data available from v19.proteinatlas.org). Furthermore, recent evidence suggests that depletion of DDX3Y results in neural differentiation defects, indicating wider tissue distribution of DDX3Y than previously assumed (Vakilian et al. 2015). Notably, DDX3Y expression is consistently lower than that of its X-linked paralog (Uhlen et al. 2015) (data available from v19.proteinatlas.org).

DDX3X and DDX3Y both encode for similar DDX3 polypeptides, a DEAD-box RNA chaperone that facilitates translation initiation on $m R N A$ s with structured 5 ' leaders (Oh etal. 2016; Calviello et al. 2021). We recently defined the set of genes that depend on DDX3X for their translation and

(C) 2021 Venkataramanan et al. This article, published in RNA, is available under a Creative Commons License (Attribution 4.0 International), as described at http://creativecommons.org/licenses/by/4.0/. 
proposed a model where this translation is facilitated by the resolution of these highly structured $5^{\prime}$ leaders by 40 -associated DDX3X (Calviello et al. 2021), consistent with prior work (Chen et al. 2018). However, the role of DDX3Y in translation and gene expression-and how similar or different its function is to DDX3X-remains incompletely understood.

Genetic diseases involving DDX3X such as DDX3X syndrome demonstrate severe sex bias. A recent cohort ( $n=107,104$ females, three males) contains de novo genetic lesions in DDX3X that are exclusively heterozygous (or hemizygous in males) (Lennox et al. 2020). These observations have been attributed to the inability of the $Y$-linked DDX3 paralog to functionally complement DDX3X, leading to embryonic lethality in males with inactivating mutations of DDX3X-whereas heterozygous females are viable, but suffer from symptoms of varying intensity. $D d x 3 y$ is unable to compensate for the loss of $D d x 3 x$ during embryonic development in mice (Chen et al. 2016). This is in contrast to the observation that mouse $D d x 3 y$ is able to compensate for the loss of $D d x 3 x$ during neural development, insulating male mice from ataxia and seizure phenotypes upon ablation of $D d x 3 x$, but is unable to reverse the susceptibility to hindbrain malignancies conferred by $D d x 3 x$ loss (Patmore et al. 2020). Conversely, the loss of DDX3Y confers infertility in human males, despite the robust expression of DDX $3 X$ in germ-line tissues (Ramathal et al. 2015). In principle, sex bias in DDX3X-dependent disorders could arise from differential expression between DDX3X and DDX3Y across tissues, different activities of their gene products, dosage compensation by the wildtype locus, or other unknown mechanisms.

In this study, we tested the functions of DDX3X and DDX3Y proteins in translation through cell-based and biochemical assays. We depleted endogenous DDX3X protein in male HCT 116 cells using an inducible degron system, complemented with either DDX3X or DDX3Y cDNAs, and measured translation and RNA abundance using ribosome profiling and RNA-seq. We find that DDX3Y or DDX3X complements DDX3X loss in protein synthesis. Using an in vitro reporter system, we demonstrate that genes that are robustly susceptible to depletion of DDX3 do not show significant changes upon substitution of DDX3X with DDX3Y. We further find that DDX3X and DDX3Y proteins have similar stability in cells. Taken together with their distinct tissue-level expression patterns, our data suggest that the $\mathrm{X}$ - and $\mathrm{Y}$-linked paralogs of DDX3 are redundant in protein synthesis, implying tissue-specific variation of DDX3Y expression or another mechanism beyond translational control underlies the sex bias of DDX3X-associated developmental disorders.

\section{RESULTS}

The DEAD-box RNA helicase DDX3 has two sex-linked paralogs. The $X$-linked DDX3X gene is located on a non- pseudoautosomal region of the short arm of the X-chromosome (Fig. 1A). DDX3Y, a paralog of DDX3X, is located on the long arm of the $Y$ chromosome in the male-specific region (Fig. 1A). The sex bias of DDX3X syndrome suggests that males with inactivating mutations in DDX3X suffer embryonic lethality, implying that human DDX3Y cannot functionally complement DDX3X. However, DDX3X and DDX3Y protein products show $\sim 92 \%$ homology, with most of the differences in the amino-terminal domain (Fig. 1B), which is dispensable for RNA duplex unwinding in vitro (Floor et al. 2016). In contrast, the amino-terminal domain of DDX3 is important for protein-protein interactions and contains a nuclear export sequence (Shih et al. 2012; Floor et al. 2016), suggesting that changes to this region in DDX3X versus DDX3Y proteins could result in functional changes in cells. A phylogenetic tree of available mammalian DDX3 protein sequences reveals significant homology between mammalian DDX3Y, and separation of the "DDX3Y clade" away from DDX3X (Fig. 1C; Supplemental Fig. S1). In many cases, DDX3Y sequences of evolutionary distant mammals show greater homology than the paralogs within a given species (Fig. 1C; Supplemental Fig. S1). However, this phylogenetic tree also suggests that DDX3X proteins are more highly conserved than DDX3Y proteins, consistent with comparatively fewer missense variants in DDX3X compared to DDX3Y in humans in the gnomAD data set (missense $Z$ score of 2.1 for DDX3Y vs. 4.33 for DDX3X [Karczewski et al. 2020]). Both DDX3X and DDX3Y are intolerant of loss-offunction alleles in the gnomAD data set. This indicates both a high degree of conservation of the DDX3 orthologs as well as a potential function and evolutionary flexibility for DDX3Y distinct from its $\mathrm{X}$-linked paralog.

In order to dissect the similarities and differences between the functions of DDX3X and DDX3Y, we used a cell line we generated to rapidly and efficiently degrade endogenous DDX3 protein $\mathrm{X}$ in human male-derived colorectal cancer HCT 116 cells, which show a stable diploid karyotype (Waldman et al. 1995; Natsume et al. 2016). We tagged DDX3X with a 68 amino acid fragment of the Auxin Inducible Degron (AID) tag termed mini-AID (mAID) in parental cells expressing the auxin-responsive F-box protein, TIR1, from Oryza sativa (OsTIR1) (Natsume et al. 2016). Cells treated with the synthetic auxin, Indole-3-acetic acid (IAA), exhibit $>90 \%$ reduction in DDX3X protein levels by $12 \mathrm{~h}$ of treatment (Fig. 2A; Calviello et al. 2021). Depletion of DDX3X (48-h auxin treatment only) affects the translation of a subset of cellular messages (Supplemental Fig. S2A). Transcripts with increased DDX3X sensitivity have low basal translation efficiency and increased GC content and RNA structure within their $5^{\prime}$ leader sequences (Supplemental Fig. S2B). This observation is consistent with previously reported results (Calviello et al. 2021).

Lentiviral transduction with either FLAG-tagged DDX3X or DDX3Y cDNAs followed by degradation of endogenous 
A

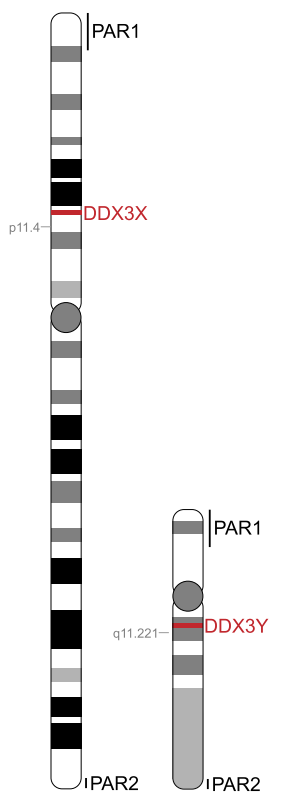

B

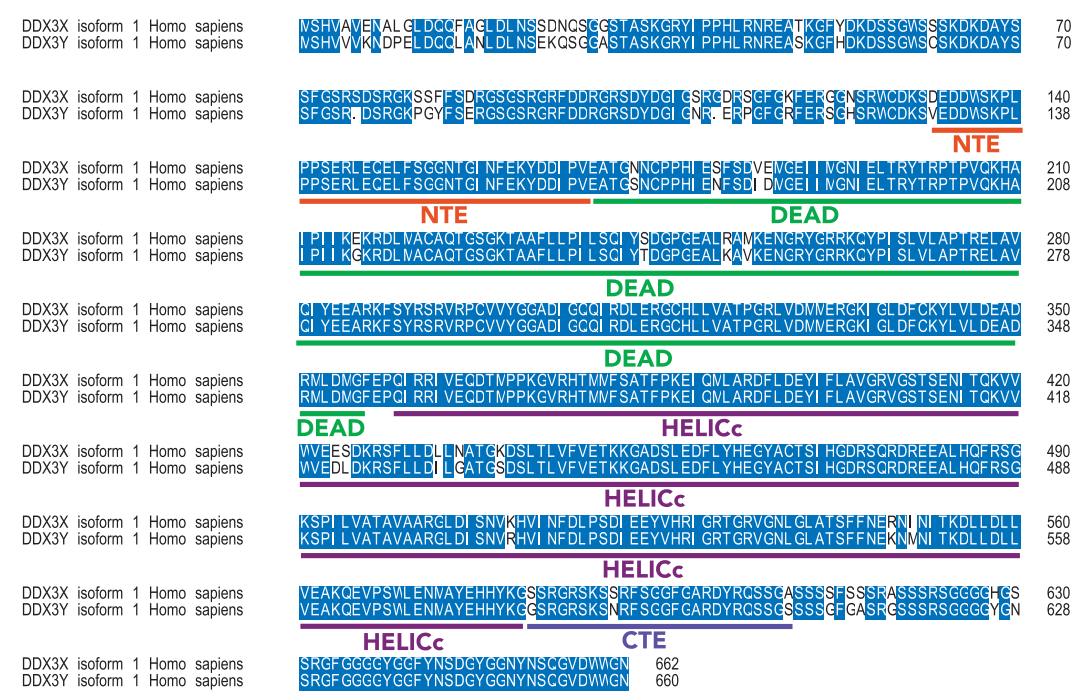

$\underset{X}{\bigotimes}$ non conserved

C

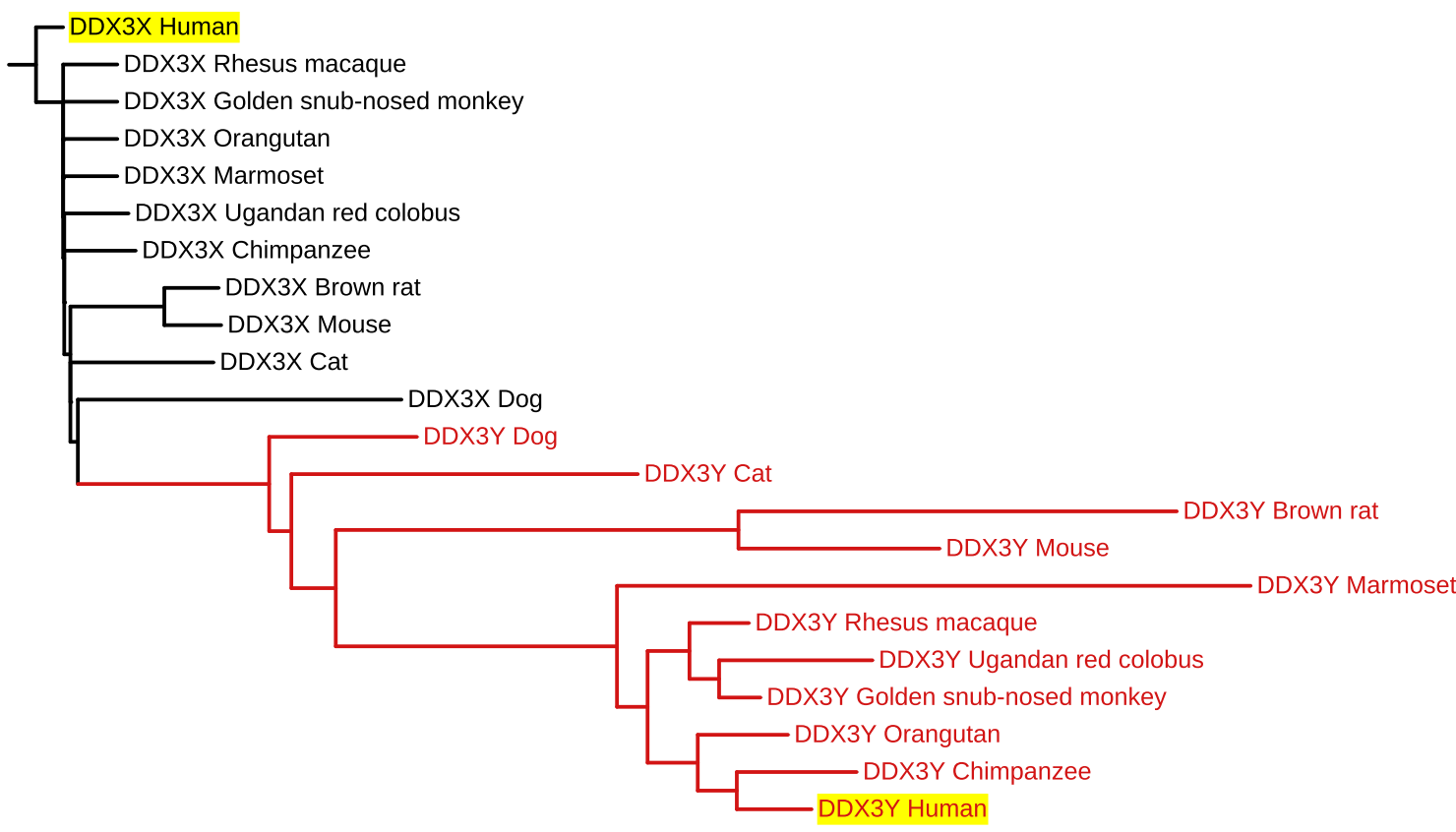

FIGURE 1. (A) Graphic indicating the locations of DDX3X and DDX3Y on their respective chromosomes. Pseudoautosomal regions (PAR) are indicated. (B) Alignment of human DDX3X and DDX3Y demonstrating 92\% sequence identity. Domain architecture of DDX3X is indicated. (C) Phylogenetic tree indicating distances between the sequences of DDX3X and DDX3Y in mammals (where sequence is available, only selected species indicated, see Supplemental Figure S1 for tree of all available mammalian sequences). The cluster of mammalian DDX3Y orthologs is indicated with red branches. Human orthologs are highlighted in yellow.

DDX3X protein allowed for almost complete replacement of the endogenous DDX3 protein with exogenous DDX3X or DDX3Y (Fig. 2B,C). We measured transcript levels and ribosome occupancy under these experimental conditions using ribosome profiling and RNA-seq. Expression of DDX3Y or DDX3X after endogenous DDX3X depletion shows similar transcript levels and ribosome densities across the transcriptome (Fig. 2D). Introduced DDX3X and DDX3Y proteins were expressed to a similar degree (Fig. 2C), but DDX3Y appeared to be more highly expressed at the RNA level (Fig. 2D) because its endogenous expression is low in HCT 116 cells (endogenous DDX3X $\mathrm{TPM}=162$; endogenous DDX3Y TPM $=0.1$; transduced DDX3X TPM $=284$; transduced DDX3Y TPM $=174$; see 
A

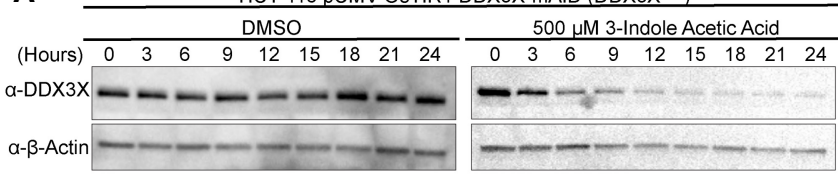

B

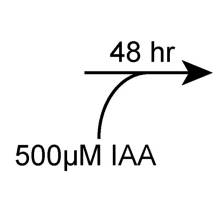

CHX treatment
Footprinting
Library Prep

Sequence

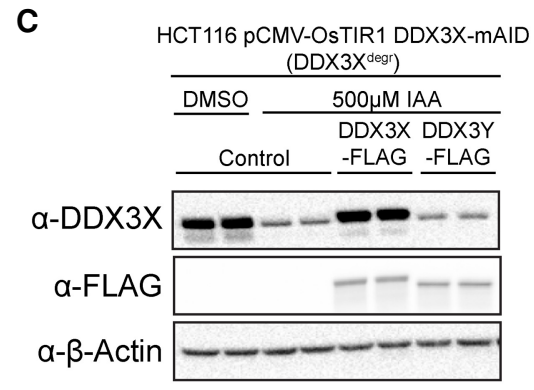

\section{D}
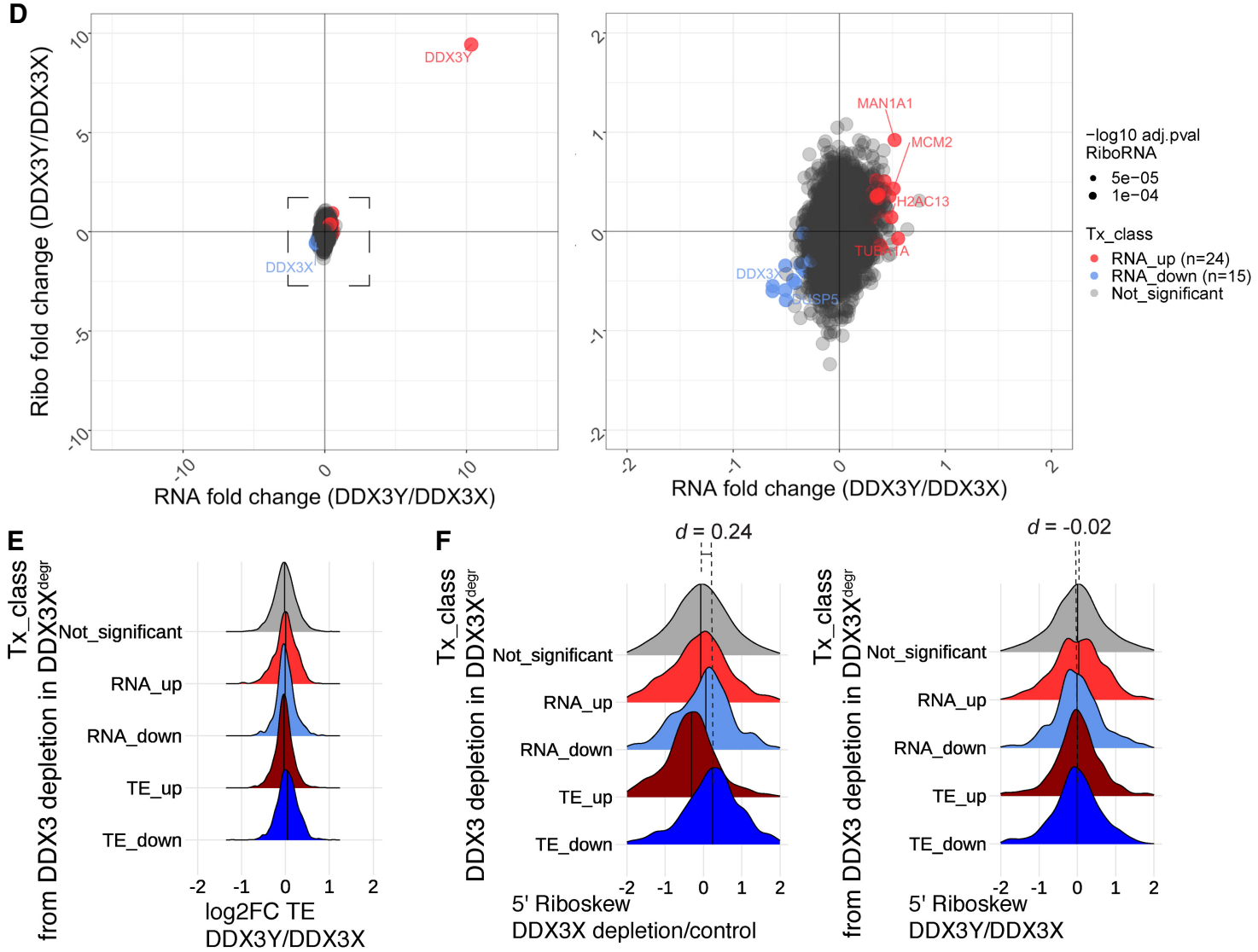

FIGURE 2. (A) Western blot for degradation of endogenous DDX3X tagged with mini-AID (mAID) upon treatment with an auxin (indole-3-acetic acid) in HCT-116 human colorectal carcinoma cell line expressing OsTIR1 under the control of a CMV promoter (DDX ${ }^{\text {degr }}$ ). (B) Experimental schematic for ribosomal profiling after replacement of endogenous DDX3X with exogenous DDX3X or 3Y. (C) Western blot for expression of exogenous FLAG-tagged DDX3X and DDX3Y in HCT-116 cells after degradation of endogenous DDX3X. (D, left) Full and (right) zoomed in plots of differential expression analysis of RNA and ribosome profiling changes upon complementation of DDX3X with either DDX3Y or DDX3X. Point size indicates $P$-value of differential translation (two biological replicates, each condition). (E) Fold-change in TE between DDX3Y and DDX3X expression in mRNAs classified based on DDX3 sensitivity (as in Supplemental Fig. S2A). (F) The fold-change of the riboskew, or ratio in ribosome occupancy in the $5^{\prime}$ UTR versus the coding sequence under DDX3 depletion (left) or the ratio between complementation with DDX3Y or DDX3X (right). Effect size (Cliff's delta) between the "Not_significant" and "TE_down" groups is indicated.

Supplemental Table S3). We found that exogenous expression of either DDX3X or DDX3Y rescued almost all changes in translation suffered due to depletion of endogenous DDX3X (99\% in DDX3X and $94 \%$ in DDX3Y) (Supplemental Fig. S2C, compare to Supplemental Figs. S2A, S3A).
To increase statistical power to detect a difference between DDX3X and DDX3Y, we tested for differences between groups of transcripts. Transcripts sensitive to depletion of DDX3X (as in Supplemental Fig. S2A) show no change in their translation efficiency upon substitution of DDX3X with DDX3Y (Fig. 2E). Additionally, DDX3X- 
sensitive transcripts show increased ribosome occupancy in $5^{\prime}$ leaders compared to the CDS (5' riboskew), consistent with previous observations (Fig. 2F; Calviello et al. 2021). However, DDX3X-sensitive transcripts show no change in 5' riboskew upon the expression of DDX3Y compared to DDX3X (Fig. 2F). Furthermore, transcripts with the largest magnitude of changes in TE between DDX3Y and DDX3X expression show no propensity for increased $5^{\prime}$ UTR GC content, a signature of DDX3 sensitivity (Supplemental Fig. S2D). The small minority of transcripts whose translation was incompletely rescued by exogenous DDX3 paralog expression also show no change in $5^{\prime}$ riboskew-a characteristic of DDX3-sensitive transcripts (Supplemental Fig. S2E). These observations are consistent with the $X$ - and $Y$-linked paralogs of DDX3 performing redundant functions in translation.

We sought to validate the impact of DDX3X depletion on the translation of selected targets and their rescue upon complementation by either DDX3X or DDX3Y. We selected candidates for DDX3 susceptibility (DVL2 long and short isoforms, ODC1, and PRKRA) and controls (ATF5, CCNE1, $R P L P 1$, and SIKE1) based on a combination of their susceptibility to DDX3 depletion in this and previous studies (Fig. 3A; Supplemental Fig. S3B), previously measured half-lives and availability of antibodies. Ribosome profiling data provide a snapshot of ribosome engagement, and acute changes in ribosome occupancy may or may not result in changes in steady-state protein levels. Therefore, we starved cells of methionine for $24 \mathrm{~h}$ to impede translation and allow for clearance of a fraction of previously synthesized proteins by the cellular degradation machinery. We followed by reintroducing complete media to restart protein synthesis and measured protein levels of our candidates by western blot (Fig. 3B). We observe that the protein levels of DDX3 target genes, but not control genes, are reduced upon depletion of DDX3X, and restored upon complementation with either DDX3X or DDX3Y (Fig. 3C).

In order to further test the impact of DDX3X and DDX3Y on translation, we used a set of previously described DDX3-sensitive $5^{\prime}$ UTRs upstream of a Renilla luciferase reporter and performed in vitro transcription, capping and 2'-O-methylation to generate a set of reporter RNAs (Calviello et al. 2021). We made translation extracts from HCT 116 cells either depleted of endogenous DDX3X or depleted and complemented by transient transfection of exogenous DDX3X and DDX3Y (Fig. 4A,B). As previously observed, 5' UTRs of DDX3-sensitive mRNAs also confer DDX3 dependence to a luciferase reporter, while the control RNA with a simple
A
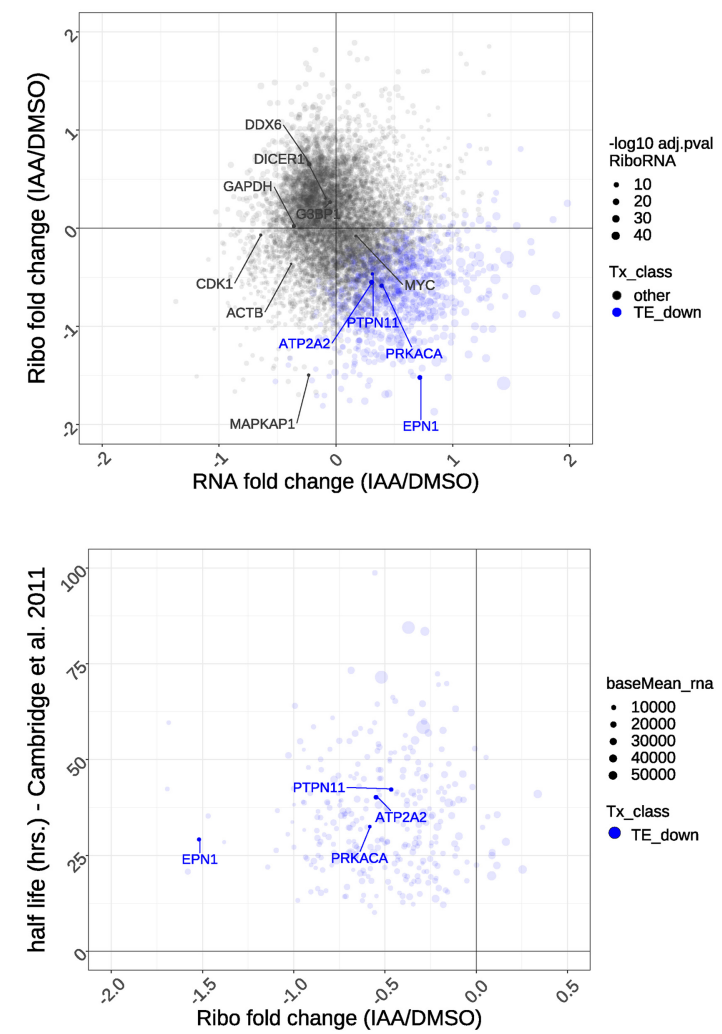

B

HCT116 cells

DDX3X-mAID
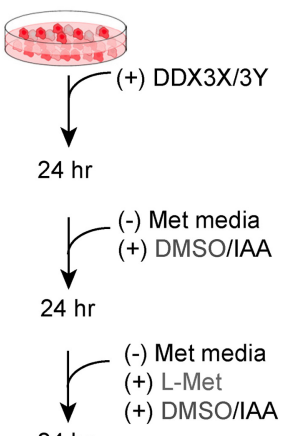

$24 \mathrm{hr}$

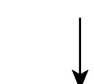

Lysis \& Western blots
C

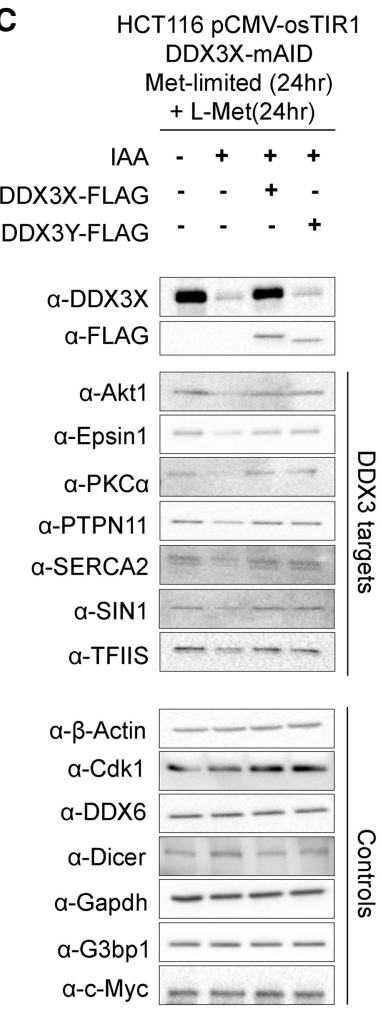

FIGURE 3. (A) Selected DDX3-sensitive transcripts are indicated by the effect of DDX3 on their translation (top) and the half-lives of their protein products (bottom). (B) Experimental schematic for Methionine-chase assay. (C) Western blot for protein products of DDX3-sensitive or control transcripts upon DDX3X depletion and complementation with DDX3X or DDX3Y. 
A

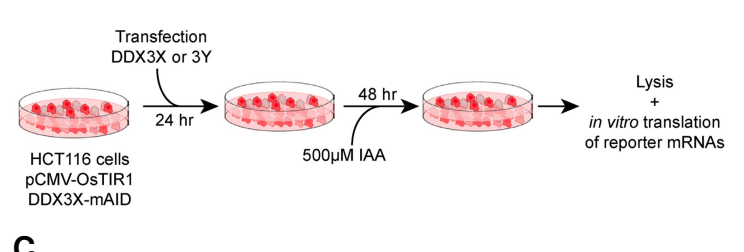

B

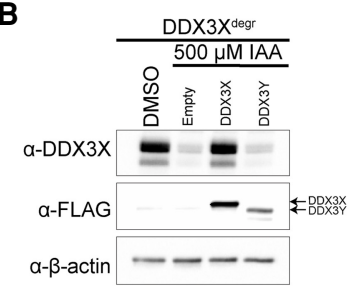

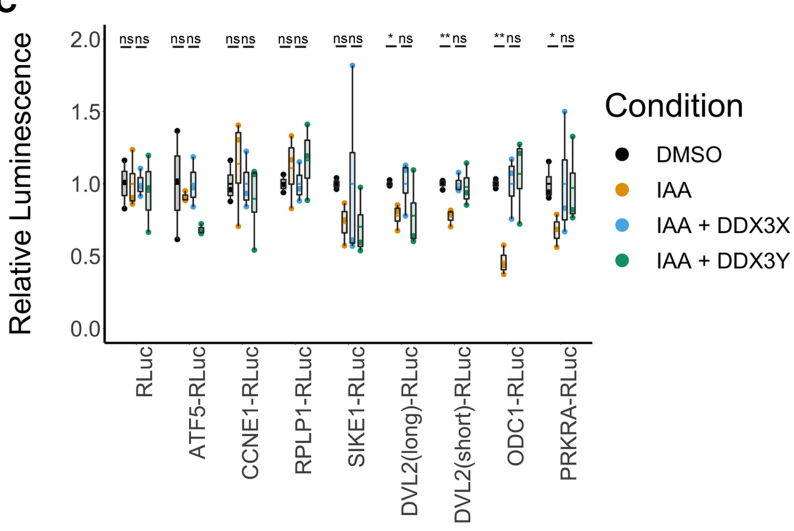

Constructs - 5'UTR Fusions

FIGURE 4. (A) Experimental schematic for in vitro translation after replacement of endogenous DDX3X with exogenous DDX3X or 3Y. (B) Western blot for degradation of endogenous DDX3X tagged with mini-AID (mAID) and expression of exogenous FLAG-tagged DDX3X and DDX3Y in HCT-116 cells after degradation of endogenous DDX3X in in vitro translation extracts. (C) Translation of in vitro transcribed reporter RNAs in lysates from control (DMSO) or DDX3X-degraded (auxin treated) HCT-116 cells as well as either FLAG-tagged DDX3X or DDX3Y on top of depletion of endogenous DDX3X (three biological replicates, each condition).

5' UTR remains unaffected (Fig. 4C). Consistent with the ribosome profiling results, DDX3-sensitive 5' UTRs show no statistically significant change in reporter output when translated with extracts containing only DDX3Y compared to DDX3X (Fig. 4C). This includes the ODC1 reporter, which is sensitive to every perturbation in DDX3X that we have tested, included depletion and inactivating mutants (Calviello et al. 2021). However, we note that the DVL2(long) reporter has a visual difference between DDX3X and DDX3Y complementation that did not reach statistical significance. These data further reinforce the notion that DDX3X and DDX3Y are functionally redundant in regulation of translation.

The results above suggest that DDX3Y can rescue translational deficiencies caused by loss of DDX3X. To reconcile this with the observed sex-bias in DDX3X-dependent disorders, we considered multiple possibilities. To eliminate any role of differences in relative protein degradation rates, we examined the stability of DDX3X or DDX3Y proteins in HEK293T cells by treatment with cycloheximide (CHX). We observed rapid loss of c-Myc but no measurable degradation of either paralog before the onset of severe $\mathrm{CHX}$ toxicity (2d, Fig. 5A). Therefore, even if DDX3Y is more or less stable than DDX3X on a longer timescale than $2 \mathrm{~d}$, both are still very stable proteins. Next, we examined FANTOM-CAGE expression data for the paralogs across multiple human tissues (Lizio et al. 2015). Our examination confirmed that expression of DDX3Y is prominent in the tissues of the male reproductive system (prostate, seminal vesicle, testes etc.; Fig. 5B). Furthermore, DDX3Y expression is undetectable in several cell types in the brain, including the midbrain, hippocampus, thalamus and amygdala (Fig. 5B). Taken together, we found that DDX3X and DDX3Y functioned similarly in translation and are differentially expressed at the RNA level between human tissues.

\section{DISCUSSION}

The DEAD-box protein DDX3 is encoded by two paralogous genes in humans, DDX3X and DDX3Y, the protein products of which show $92 \%$ sequence identity (Fig. 1B). Heterozygous inactivating mutations in DDX3X are linked to DDX3X syndrome, a neurodevelopmental delay and autism-spectrum disorder (Lennox et al. 2020). DDX3X syndrome shows a severe sex bias with $>95 \%$ of known cases affecting females-indicating that DDX3Y is unable to compensate for the impairment of DDX3X function in brain despite recent observations that DDX3Y may also modulate neural development (Vakilian et al. 2015). Conversely, loss of DDX3Y results in a male infertility phenotype despite robust expression of DDX3X in male germline tissues, indicating that DDX $3 X$ cannot complement loss of DDX3Y in the male reproductive system (Rauschendorf et al. 2014; Ramathal et al. 2015; Kotov et al. 2017)

In this and previous work, we have demonstrated using orthogonal systems that DDX3X is required for translation initiation on transcripts with structured $5^{\prime}$ leader sequences (Supplemental Fig. S2A,B; Calviello et al. 2021). In this study, we examined the global translation profiles when cells depleted of endogenous DDX3X are complemented with CDNAs of either DDX3X or DDX3Y. We could not detect significant changes of translation or steady state RNA levels (Fig. 2). We used in vitro translation experiments with a luciferase reporter to confirm 5 leaders of genes that are sensitive to either depletion of DDX3X or inactivating mutations show no significant differences in translation in extracts containing either DDX3X or DDX3Y (Fig. 4). In fact, the genes that show decreased translation (TE_down) upon depletion of DDX3X show an increased change in the relative ribosome occupancy in 
the $5^{\prime}$ leader compared to the CDS, a signature that is lost while comparing DDX3Y to DDX3X (Fig. 2; Supplemental Fig. S2). This suggests that the two human paralogs of DDX3 perform equivalent functions in translation, at least in the context of our experimental system.

At least six models could potentially explain the inability of the DDX3 paralogs to complement each other. First, the two paralogs of DDX3 could have distinct target complements and affect the translation of different sets of transcripts. Second, the two paralogs could have the same target propensity but distinct subcellular localizations, and thus differ in their effects. These possibilities are partially rebuffed by the gain of DDX3Y expression complementing for loss of DDX3X in certain lymphoid malignancies (Gong et al. 2021) and in BHK21 hamster cells (Sekiguchi et al. 2004). Additionally, a genome-wide CRISPR screen for essential genes identified DDX3Y as being essential only in Raji cells (which are also of hematopoietic origin), which had suffered a truncating mutation in DDX3X, further indicating genetic complementation (Wang et al. 2015). However, genetic complementation might mask a subtle fitness defect or gene expression changes that could be important in other cellular states. Third, the two differences in protein sequences between the paralogs could lead to differential stability and residence time in the cell. In this study, we quantitatively demonstrate that the translation profile of DDX3X and DDX3Yexpressing cells are functionally identical, and that the paralogs are both exceptionally long-lived in cells, arguing against the three scenarios laid out above.

A fourth model to explain the lack of paralog complementation at the organism level is tissue-specific expression patterns of the paralogs. At the RNA level, DDX3Y expression is sporadic in the different cell types of the brain, as well as all tissues excepting those of the male reproductive system (Fig. 5). It has previously been suggested that specific transcriptional and translational regulation contribute to increased DDX3Y protein levels in the testes (Jaroszynski et al. 2011; Rauschendorf et al. 2011). One potential explanation for the inability of DDX3Y to complement DDX3X in the brain could be that the protein is expressed in insufficient quantities. Conversely, although DDX3X is detectable in adult male testes, multiple layers of transcriptional and translational control restrict its expression to post-meiotic spermatids (Rauschendorf et al. 2014). The absence of DDX3X protein in premeiotic spermatids could explain its inability to rescue the infertility phenotype conferred by DDX3Y loss. A recent analysis of tissue-specific expression of sex-linked paralogs correcting for multimapping short sequencing reads estimated that while the cumulative expression of DDX3 is statistically identical across tissues for individuals with $X X$ and $X Y$ genotypes, the DDX3Y expression makes up only a small minority of total DDX3 (Godfrey et al. 2020). This lends further support to the idea that while the two paralogs are functionally identical, spatiotemporal differences in their expression precludes complementation in a number of scenarios. It is important to note that this model requires future work to be confirmed. Lineage-specific complementation of DDX3 paralogs in vivo would formally test this hypothesis, and there are already hints of paralog-specific developmental effects (Patmore et al. 2020).

Two models remain that cannot be formally ruled out. First, there could be context-dependent factors in neurodevelopment and germ-cell development that cause differential translational regulation by the $\mathrm{X}$ - and $\mathrm{Y}$ - linked DDX3 paralogs, and that these contribute to their inability to complement each other in these contexts-despite the gene products of DDX3X and DDX3Y having redundant functions. Most of the differences in the polypeptide sequences of DDX3X and DDX3Y are in the amino- and carboxy-terminal domains, outside the helicase core, that are sites of protein-protein interactions. It is conceivable that these differences might drive differences in the interactomes of the paralogs. Some evidence in support of this model comes from the observation that in mice, while $D d x 3 y$ is able to compensate for the neurodevelopmental phenotypes caused by the loss of $D d x 3 x$ in neural lineages, it is unable to suppress the increase in hindbrain malignancies also caused by Ddx3x loss (Patmore et al. 2020). This would indicate some form of context-dependent accessory factor influencing the target complement of Ddx3 homologs. However, it is worth noting that the ability of male mice with genetic lesions in $D d x 3 x$ to survive without neurological anomalies has not been recapitulated in humans as genetic variation in DDX3X in the human population is extremely low (Karczewski et al. 2020). Lastly, it is formally possible that there are mechanisms outside protein synthesis that both DDX3X and DDX3Y contribute to and that they function differently in those other functions. This work focused on the role of DDX3X and DDX3Y in protein synthesis, where data show they function redundantly, but the marginal differential expression that persists after complementation could be the result of functions outside protein synthesis (Supplemental Fig. S2C).

We suggest that some combination of tissue-specific paralog and unknown cofactor expression may explain the sex bias of DDX3X-associated developmental disorders, but that inherent differences in molecular function are unlikely to drive this phenomenon. Further investigations in these directions could reveal specific targets or factors that could be exploited to mitigate the phenotypic consequences of genetic lesions in the DDX3 paralogs.

\section{MATERIALS AND METHODS}

\section{DDX3 sequence alignment and phylogenetic tree}

Reference protein sequences of human DDX3X and DDX3Y were obtained from RefSeq, and alignment was performed using the 
A A Transfected HEK-293T Cells

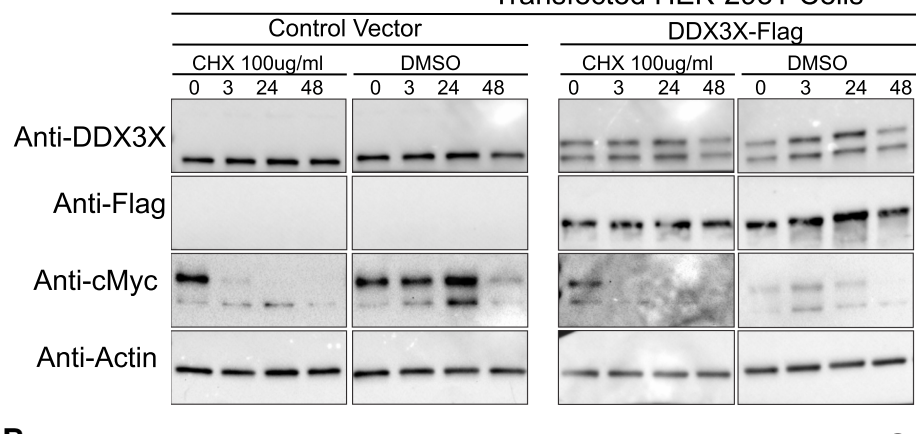

B

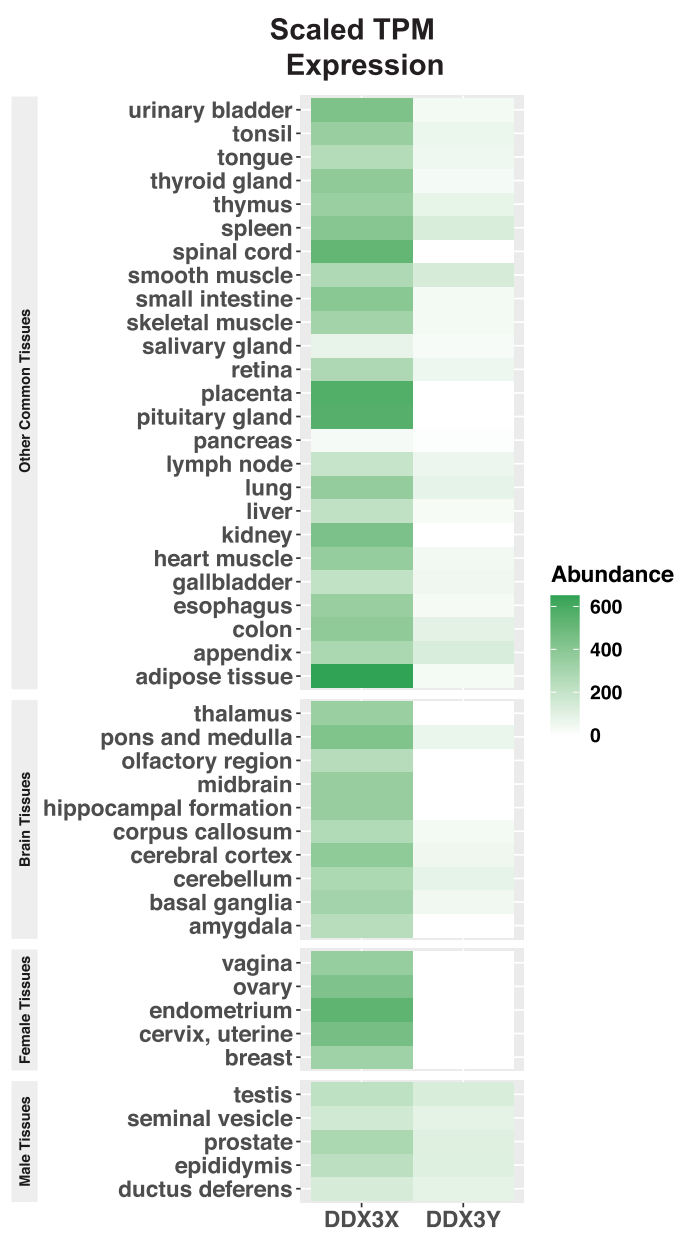

Quartile normalized Expression

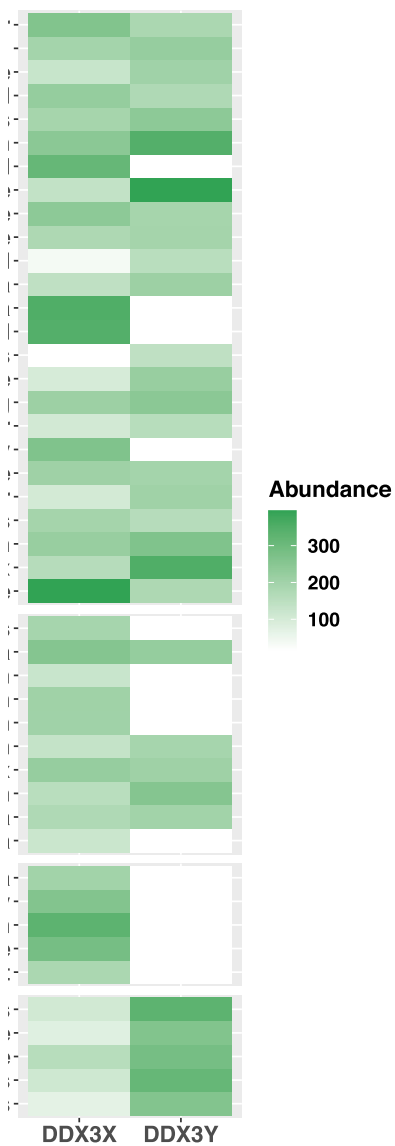

FIGURE 5. (A) Western blots of endogenous and transfected DDX3 paralogs (DDX3X and DDX3Y) for $48 \mathrm{~h}$ after treatment with cycloheximide $(100 \mu \mathrm{g} / \mathrm{mL})$. Myc is used as a control for rapid degradation. ( $B$, left) Scaled TPM expression of DDX3X and DDX3Y across a number of human tissues (data from GTeX). (Right) Quartile-normalized expression of DDX3X and DDX3Y across a number of human tissues (data from GTeX). DDX3Y expresson is particularly prominent in male-specific tissue types such as prostate and seminal vesicles, in the testes, and notably deplete in numerous tissues of the central nervous system.

MAFFT algorithm using the BLOSUM62 scoring matrix with default parameters (gap open penalty $=1.53$; gap extension penalty $=0.123$ ) (Katoh et al. 2002). Protein sequences for mammalian DDX3 orthologs (both $X$ and $Y$, where available and reliably annotated) were obtained using a combination of the NCBI Gene ortholog finder and PSI-blast (Altschul et al. 1997). Multiple sequence alignment was performed as described above, and a phy- logenetic tree was constructed using the Interactive Tree of Life (iTOL) tool (v5.6.3) (Letunic and Bork 2019).

\section{Cell line construction}

HCT-116 cells expressing OsTIR1 were constructed as described in Natsume et al. (2016). Briefly, a plasmid coexpressing gRNA for 
the AAVS1 safe-harbor locus and SpCas9 was cotransfected with plasmid encoding for OsTIR1 and homologous arms for the HDR at the AAVS1 locus. Selection was performed in $1 \mu \mathrm{g} / \mathrm{mL}$ of puromycin; colonies were picked and validated by PCR and western blot.

The HCT-116 OsTIR1 expressing cell line was transfected with $800 \mathrm{ng}$ (total) of an equimolar mixture of pX330-based CRISPR/ Cas plasmid with guide RNAs targeting the carboxy-terminal end of DDX3X and 1200 ng of a short homology donor carrying the mAID degron tag with a G418 resistance cassette in a pUC19 backbone. Selection was performed in $700 \mu \mathrm{g} / \mathrm{mL}$ of G418, and single colonies picked and validated by PCR and western blot. Selection, picking, clonal expansion, and validation were repeated twice (total of three rounds) to ensure a clonal cell line.

Lentiviral constructs expressing FLAG-tagged and partially codon-optimized DDX3X or DDX3Y (ORFs obtained from Twist Biosciences, cloned upstream of tandem P2A cleavage site followed by mCherry2) were transfected into HEK293T cells along with packaging plasmids. Three days post transfection, the media containing viral particles were harvested and filtered, before being used for infection of the DDX3X-degron-containing cell line described above. Cells with successful expression of DDX3 paralogs were isolated by sorting for $\mathrm{mCherry} 2$ positive cells. Three rounds of sorting were performed to ensure stable expression. Levels of DDX3X or DDX3Y protein were confirmed by western blot against the FLAG epitope.

\section{Induction of DDX3X degradation}

For ribosome profiling, $500 \mu \mathrm{M}$ Indole-3-acetic acid (IAA, Grainger, 31FY95) was added to the cells (in fresh media) from a $500 \mathrm{mM}$ stock in DMSO. For in vitro translation lysates, CMVpromoter expression plasmids $(50 \mu \mathrm{g}$ of DDX3X-FLAG and 75 $\mu \mathrm{g}$ of DDX3Y-FLAG per $15 \mathrm{~cm}$ plate, to equalize expression levels) were transfected into cells using a 3:1 ratio of lipofectamine 2000 to plasmid. Twenty-four hours post-transfection, $500 \mu \mathrm{M}$ Indole-3-acetic acid (IAA, Grainger, 31FY95) was added to the cells (in fresh media) from a $500 \mathrm{mM}$ stock in DMSO. Protein levels were assayed by western blot.

\section{Antibodies}

Primary antibodies used in this study include rabbit polyclonal anti-DDX3 (custom made by Genemed Synthesis using peptide ENALGLDQQFAGLDLNSSDNQS) (Lee et al. 2008), anti-actin HRP (Santa Cruz, sc-47778), anti-FLAG HRP (Sigma, A8592), anti-Akt1 (Bethyl, A302-065A), anti-Epsin1 (Bethyl, A304-524A), anti-PKC $\alpha$ (Bethyl, A302-445A), anti-PTPN11 (Bethyl, A301544A), anti-SERCA2 (Bethyl, A300-406A), anti-Sin1 (Bethyl, A300-910A), anti-TFIIS (Bethyl, A302-240A), anti-Cdk1 (Santa Cruz, sc-54), anti-DDX6 (Novus, NB200-191), anti-Dicer (SantaCruz, sc-136979), anti-GAPDH, anti-G3BP1 (Bethyl A302-034A), and anti-cMyc (abcam, ab205818).

\section{Ribosome profiling library construction}

One $15 \mathrm{~cm}$ dish of cells at $80 \%-90 \%$ confluency was used per replicate. Twenty-four hours post plating, media were changed and fresh media with $500 \mu \mathrm{M}$ IAA were added to cells. Forty-eight hours after auxin addition, cells were treated with $100 \mu \mathrm{g} / \mathrm{mL}$ cycloheximide $(\mathrm{CHX})$ and harvested and lysed as described in McGlincy and Ingolia (2017). Briefly, cells were washed with PBS containing $100 \mu \mathrm{g} / \mathrm{mL} \mathrm{CHX}$ and lysed in ice-cold lysis buffer (20 mM TRIS-HCl pH 7.4, $150 \mathrm{mM} \mathrm{NaCl}, 5 \mathrm{mM} \mathrm{MgCl}, 1 \mathrm{mM}$ DTT, $100 \mu \mathrm{g} / \mathrm{mL}$ CHX, 1\% [v/v] Triton X-100, $25 \mathrm{U} / \mathrm{mL}$ TurboDNase [Ambion]). A total of $240 \mu \mathrm{L}$ lysate was treated with $6 \mu \mathrm{L}$ RNase I (Ambion, $100 \mathrm{U} / \mu \mathrm{L}$ ) for $45 \mathrm{~min}$ at RT with gentle agitation and further digestion halted by addition of SUPERase $\bullet$ In (Ambion). Illustra Microspin Columns S-400 HR (GE Healthcare) were used to enrich for monosomes, and RNA was extracted from the flow-through using a Direct-zol Kit (Zymo Research). Gel slices of nucleic acids between 24-32 nt long were excised from a 15\% urea-PAGE gel. Eluted RNA was treated with T4 PNK and preadenylated linker was ligated to the $3^{\prime}$ end using T4 RNA Ligase 2 truncated KQ (NEB, M0373L). Linker-ligated footprints were reverse transcribed using Superscript III (Invitrogen) and gel-purified RT products circularized using CircLigase II (Lucigen, CL4115K). rRNA depletion was performed using biotinylated oligos as described in Ingolia et al. (2012) and libraries constructed using a different reverse indexing primer for each sample.

RNA was extracted from $25 \mu \mathrm{L}$ intact lysate (nondigested) using the Direct-zol Kit (Zymo Research), and stranded total RNA libraries were prepared using the TruSeq Stranded Total RNA Human/ Mouse/Rat Kit (Illumina), following manufacturer's instructions.

Libraries were quantified and checked for quality using a Qubit fluorimeter and Bioanalyzer (Agilent) and sequenced on a HiSeq 4000 sequencing system (single end, 65 nt reads).

\section{Preprocessing and alignment of NGS data}

All NGS data were processed as previously described (Calviello et al. 2021). Briefly, adapter sequences were trimmed from the footprint reads, UMI sequences were collapsed and removed. Reads aligned to rRNA or a collection of snoRNAs, tRNAs, and miRNAs were discarded. The filtered reads were mapped to the hg38 version of the human genome using STAR (Dobin et al. 2013) and count matrices built as described previously (Calviello et al. 2021) using Ribo-seQC (Calviello et al. 2019). Only reads above $24 \mathrm{nt}$ long were counted. Read counts for all libraries are in Supplemental Table S1.

\section{Differential expression analysis}

Differentially expressed (DE) genes between the samples were identified using DESeq2 (Love et al. 2014). Only genes with average normalized number of counts greater than 20 for both RNA and Ribo-seq were retained. Genes with transcript levels were defined as either "RNA_up" or "RNA_down," with an adjusted $P$-value cutoff of 0.01 . Differential translation regulation was calculated using DESeq2 interaction terms and a likelihood ratio test, with an adjusted $P$-value cutoff of 0.05 , and differentially translated genes were defined as "TE-up" or "TE_down." DE details for all genes that met the read count cutoff are in Supplemental Table S2. 


\section{Transcript features analysis}

$5^{\prime}$ UTR ribosome skew (riboskew) was calculated as previously defined (Calviello et al. 2021). Effect sizes (nonparametric Cliff's Delta measure) between the transcript classes were calculated using the "effsize" R package (Torchiano 2020).

\section{Methionine starvation and chase}

Candidates for validation of ribosome profiling were chosen based on effects of DDX3X depletion of translation efficiency, measured half-lives (Cambridge et al. 2011), and availability of antibodies. Expression plasmids carrying DDX3X-FLAG or DDX3YFLAG were transfected into cells using a 3:1 ratio of lipofectamine 2000 to plasmid. Twenty-four hours post-transfection, cells were washed three times with PBS to eliminate residual methionine. A total of $500 \mu \mathrm{M}$ Indole-3-acetic acid (IAA, Grainger, 31FY95) was added to the cells in fresh media lacking methionine from a $500 \mathrm{mM}$ stock in DMSO. Cells were maintained in methionine deficient media for $24 \mathrm{~h}$, following which media were replaced with complete media (containing IAA or DMSO). Cells were harvested $24 \mathrm{~h}$ later and lysed for western blot analysis.

\section{In vitro transcription, capping, and 2'-O-methylation of reporter RNAs}

Annotated 5' UTRs for selected transcripts were cloned upstream of Renilla Luciferase (RLuc) under the control of a T7 promoter, with 60 adenosine nucleotides downstream from the stop codon to mimic polyadenylation. Untranslated regions were cloned using synthetic DNA (Integrated DNA Technologies) or by isolation using 5' RACE (RLM-RACE Kit, Invitrogen). Template was PCR amplified using Phusion polymerase from the plasmids using the following primers, and gel purified, as described (Floor and Doudna 2016).

\section{pA60 txn rev: TTT TTT TTT TTT TTT TTT TTT TTT TTT TTT TTT TTT TTT TTT TTT TTT TTT TTT TTT TTT CTG CAG pA60 txn fwd: CGG CCA GTG AAT TCG AGC TCT AAT ACG ACT CAC TAT AGG}

A total of $100 \mu \mathrm{L}$ in vitro transcription reactions were set up at room temperature with 1-5 $\mathrm{\mu g}$ of purified template, $7.5 \mathrm{mM}$ ACGU ribonucleotides, $30 \mathrm{mM}$ Tris- $\mathrm{Cl} \mathrm{pH} 8.1,125 \mathrm{mM} \mathrm{MgCl}$, $0.01 \%$ Triton X-100, 2 mM spermidine, 110 mM DTT, T7 polymerase, and $0.2 \mathrm{U} / \mu \mathrm{L}$ of Superase $\bullet$ In RNase inhibitor (Thermo Fisher Scientific). Transcription reactions were incubated in a PCR block at $37^{\circ} \mathrm{C}$ for $1 \mathrm{~h}$. A total of $1 \mu \mathrm{L}$ of $1 \mathrm{mg} / \mathrm{mL}$ pyrophosphatase (Roche) was added to each reaction, and the reactions were subsequently incubated in a PCR block at $37^{\circ} \mathrm{C}$ for $3 \mathrm{~h}$. One unit of RQ1 RNase-free DNase (Promega) was added to each reaction followed by further incubation for $30 \mathrm{~min}$. RNA was precipitated by the addition of $200 \mu \mathrm{L} 0.3 \mathrm{M} \mathrm{NaOAc} \mathrm{pH} 5.3,15 \mu \mathrm{g}$ GlycoBlue coprecipitant (Thermo Fisher Scientific), and $750 \mu \mathrm{L}$ $100 \% \mathrm{EtOH}$. Precipitated RNA was further purified over the RNA Clean \& Concentrator-25 columns (Zymo Research). Glyoxal gel was run to assess the integrity of the RNA before subsequent capping and 2'-O-methylation.
An amount of $20 \mu \mathrm{g}$ of total RNA was used in a $40 \mu \mathrm{L}$ capping reaction with $0.5 \mathrm{mM}$ GTP, $0.2 \mathrm{mM}$ S-adenosylmethionine (SAM), 20 units of Vaccinia capping enzyme (New England Biolabs), 100 units of 2'-O-methyltransferase (New England Biolabs), and 25 units RNasin Plus RNase Inhibitor (Promega). The reactions were incubated at $37^{\circ} \mathrm{C}$ for $1 \mathrm{~h}$, followed by purification over the RNA Clean \& Concentrator-25 columns (Zymo Research) and elution in DEPC H2O. Glyoxal gel was run to assess the integrity of the RNA before proceeding to in vitro translation reactions.

\section{Preparation of cellular extracts for in vitro translation}

Three $150 \mathrm{~mm}$ plates of HCT 116 cells were trypsinized and pelleted at $1000 \mathrm{~g}, 4^{\circ} \mathrm{C}$. One cell-pellet volume of lysis buffer $(10 \mathrm{mM}$ HEPES, pH 7.5, $10 \mathrm{mM} \mathrm{KOAc}, 0.5 \mathrm{mM} \mathrm{MgOAc} 2,5 \mathrm{mM}$ DTT, and one tablet miniComplete EDTA free protease inhibitor [Roche] per $10 \mathrm{~mL}$ ) was added to the cell pellet and was incubated on ice for $45 \mathrm{~min}$. The pellet was homogenized by trituration through a 26G needle attached to a $1 \mathrm{~mL}$ syringe 13-15 times. Efficiency of disruption was checked by trypan blue staining (>95\% disruption target). The lysate was cleared by centrifugation at $14,000 \mathrm{~g}$ for $1 \mathrm{~min}$ at $4^{\circ} \mathrm{C}, 2-5 \mu \mathrm{L}$ was reserved for western blot analysis, and the remainder was aliquoted and flash frozen in liquid nitrogen. Preparation of HEK293T lysates with DDX3X siRNA knockdown and mutant expression has been previously described (Lennox et al. 2020)

\section{In vitro translation}

A total of $5 \mu \mathrm{L}$ in vitro translation reactions were set up with $2.5 \mu \mathrm{L}$ of lysate and $20 \mathrm{ng}$ total RNA (0.84 mM ATP, $0.21 \mathrm{mM}$ GTP, 21 $\mathrm{mM}$ Creatine Phosphate, 0.009 units $/ \mathrm{mL}$ Creatine phosphokinase, $10 \mathrm{mM}$ HEPES $\mathrm{pH}$ 7.5, 2 mM DTT, 2 mM MgOAc, 100 $\mathrm{mM} \mathrm{KOAc}, 0.008 \mathrm{mM}$ amino acids, $0.25 \mathrm{mM}$ spermidine, 5 units RNasin Plus RNase inhibitor [Promega]) as described by Lee et al. (2015). Reaction tubes were incubated at $30^{\circ} \mathrm{C}$ for $45 \mathrm{~min}$, and expression of the reporter was measured using the Renilla Luciferase Assay System (Promega) on a GloMax Explorer Plate Reader (Promega).

\section{Analysis of DDX3 paralog stability}

One $10 \mathrm{~cm}$ dish of HEK-293T cells at $~ 80 \%$ confluency was used per replicate. Twenty-four hours post plating, cells were transfected with a DDX3X-FLAG, DDX3Y-FLAG, or empty vector using the TransIT-LT1 Transfection Reagent System (Mirus). Plates were split into a 12 well plate $24 \mathrm{~h}$ post transfection. Twenty-four hours after resting, cells were collected for a $\mathrm{O}$ h sample. The remaining cells were treated with either $100 \mu \mathrm{g} / \mathrm{mL}$ cycloheximide $(\mathrm{CHX})$ or DMSO and collected and flash frozen in liquid nitrogen 3, 24, and $48 \mathrm{~h}$ post treatment. Samples were thawed, lysed in buffer (150 $\mathrm{mM} \mathrm{NaCl}$, 1\% IGEPAL, $50 \mathrm{mM}$ Tris- $\mathrm{HCl} \mathrm{pH}$ 8.0), homogenized, and centrifuged. The supernatant was saved for western blot analysis. 


\section{Analysis of FANTOM5 tissue level expression data}

FANTOM5 per gene transcript expression levels (CAGE data) was obtained based on The Human Protein Atlas version 19.3 and Ensembl version 92.38 (Lizio et al. 2015). Quantile-normalized and scaled TPMs were plotted for common tissues as well as male and female-specific tissues.

\section{DATA DEPOSITION}

Sequencing data can be retrieved using GEO accession number GSE180669. Source code for the analysis of ribosome profiling data was adapted from https://github.com/lcalviell/DDX3X_ RPCLIP. Source code specific to the analysis in this manuscript is available at https://github.com/srivats-venkat/DDX3X_3Y_ comparison.

\section{SUPPLEMENTAL MATERIAL}

Supplemental material is available for this article.

\section{COMPETING INTEREST STATEMENT}

S.N.F. consults for MOMA Therapeutics.

\section{ACKNOWLEDGMENTS}

We thank members of the Floor laboratory for feedback on the manuscript. Computation was supported by the UCSF Wynton computing infrastructure. This work was supported by the UCSF Program for Breakthrough Biomedical Research, funded in part by the Sandler Foundation (to S.N.F.), the California TobaccoRelated Disease Research Grants Program 27KT-0003 (to S.N. F.), the National Institutes of Health DP2GM132932 and R01NS120667 (to S.N.F.), the National Institutes of Health F32GM133144 (to S.V.), and the California Tobacco-Related Disease Research Grants Program T30DT1004 (to K.W.).

Author contributions: Conceptualization, S.V. and S.N.F.; investigation, S.V., M.G. and L.C.; writing-original draft, S.V.; writing -review and editing, S.N.F., S.V., and L.C.; resources, S.V. and K.W.; funding acquisition, S.N.F.; supervision, S.N.F.

Received July 23, 2021; accepted September 12, 2021.

\section{REFERENCES}

Altschul SF, Madden TL, Schaffer AA, Zhang J, Zhang Z, Miller W, Lipman DJ. 1997. Gapped BLAST and PSI-BLAST: a new generation of protein database search programs. Nucleic Acids Res 25: 3389-3402. doi:10.1093/nar/25.17.3389

Calviello L, Sydow D, Harnett D, Ohler U. 2019. Ribo-seQC: comprehensive analysis of cytoplasmic and organellar ribosome profiling data. bioRxiv doi:10.1101/601468

Calviello L, Venkataramanan S, Rogowski KJ, Wyler E, Wilkins K, Tejura M, Thai B, Krol J, Filipowicz W, Landthaler M, et al. 2021. DDX3 depletion represses translation of mRNAs with complex $5^{\prime}$ UTRs. Nucleic Acids Res 49: 5336-5350. doi:10.1093/nar/ gkab287
Cambridge SB, Gnad F, Nguyen C, Bermejo JL, Kruger M, Mann M. 2011. Systems-wide proteomic analysis in mammalian cells reveals conserved, functional protein turnover. J Proteome Res 10: 52755284. doi:10.1021/pr101183k

Chen CY, Chan CH, Chen CM, Tsai YS, Tsai TY, Wu Lee YH, You LR. 2016. Targeted inactivation of murine $D d x 3 x$ : essential roles of Ddx3x in placentation and embryogenesis. Hum Mol Genet 25: 2905-2922.

Chen HH, Yu HI, Yang MH, Tarn WY. 2018. DDX3 activates CBC-elF3mediated translation of uORF-containing oncogenic mRNAs to promote metastasis in HNSCC. Cancer Res 78: 4512-4523. doi:10.1158/0008-5472.CAN-18-0282

Cotton A, Price E, Jones M, Balaton B, Kobor M, Brown C. 2015. Landscape of DNA methylation on the $X$ chromosome reflects CpG density, functional chromatin state and X-chromosome inactivation. Hum Mol Genet 24: 1528-1539. doi:10.1093/hmg/ ddu564

Ditton HJ, Zimmer J, Kamp C, Rajpert-De Meyts E, Vogt PH. 2004. The AZFa gene DBY (DDX3Y) is widely transcribed but the protein is limited to the male germ cells by translation control. Hum Mol Genet 13: 2333-2341. doi:10.1093/hmg/ddh240

Dobin A, Davis CA, Schlesinger F, Drenkow J, Zaleski C, Jha S, Batut P, Chaisson M, Gingeras TR. 2013. STAR: ultrafast universal RNA-seq aligner. Bioinformatics 29: 15-21. doi:10.1093/bioinformatics/ bts635

Floor SN, Doudna JA. 2016. Tunable protein synthesis by transcript isoforms in human cells. Elife 5: e10921. doi:10.7554/eLife.10921

Floor SN, Condon KJ, Sharma D, Jankowsky E, Doudna JA. 2016. Autoinhibitory interdomain interactions and subfamily-specific extensions redefine the catalytic core of the human DEAD-box protein DDX3. J Biol Chem 291: 2412-2421. doi:10.1074/jbc.M115 .700625

Godfrey AK, Naqvi S, Chmatal L, Chick JM, Mitchell RN, Gygi SP, Skaletsky H, Page DC. 2020. Quantitative analysis of YChromosome gene expression across 36 human tissues. Genome Res 30: 860-873. doi:10.1101/gr.261248.120

Gong C, Krupka J, Gao J, Grigoropoulos NF, Screen M, Usheva Z, Cucco F, Barrans S, Painter D, Mohammed M, et al. 2021. Sequential inverse dysregulation of the RNA helicases DDX3X and DDX3Y facilitates MYC-driven lymphomagenesis. Mol Cell doi:10.1016/j.molcel.2021.07.041

Ingolia NT, Brar GA, Rouskin S, McGeachy AM, Weissman JS. 2012. The ribosome profiling strategy for monitoring translation in vivo by deep sequencing of ribosome-protected mRNA fragments. Nat Protoc 7: 1534-1550. doi:10.1038/nprot.2012.086

lossifov I, O'Roak BJ, Sanders SJ, Ronemus M, Krumm N, Levy D, Stessman HA, Witherspoon KT, Vives L, Patterson KE, et al. 2014. The contribution of de novo coding mutations to autism spectrum disorder. Nature 515: 216-221. doi:10.1038/ nature 13908

Jaroszynski L, Zimmer J, Fietz D, Bergmann M, Kliesch S, Vogt PH. 2011. Translational control of the AZFa gene DDX3Y by 5'UTR exon-T extension. Int J Androl 34: 313-326. doi:10.1111/j.13652605.2010.01079.x

Karczewski KJ, Francioli LC, Tiao G, Cummings BB, Alfoldi J, Wang Q, Collins RL, Laricchia KM, Ganna A, Birnbaum DP, et al. 2020. The mutational constraint spectrum quantified from variation in 141,456 humans. Nature 581: 434-443. doi:10.1038/s41586020-2308-7

Katoh K, Misawa K, Kuma K, Miyata T. 2002. MAFFT: a novel method for rapid multiple sequence alignment based on fast Fourier transform. Nucleic Acids Res 30: 3059-3066. doi:10.1093/nar/gkf436

Kool M, Jones DT, Jager N, Northcott PA, Pugh TJ, Hovestadt V, Piro RM, Esparza LA, Markant SL, Remke M, et al. 2014. Genome sequencing of $\mathrm{SHH}$ medulloblastoma predicts 
genotype-related response to smoothened inhibition. Cancer Cell 25: 393-405. doi:10.1016/j.ccr.2014.02.004

Kotov AA, Olenkina OM, Godneeva BK, Adashev VE, Olenina LV. 2017. Progress in understanding the molecular functions of DDX3Y (DBY) in male germ cell development and maintenance. Biosci Trends 11: 46-53. doi:10.5582/bst.2016.01216

Lee CS, Dias AP, Jedrychowski M, Patel AH, Hsu JL, Reed R. 2008. Human DDX3 functions in translation and interacts with the translation initiation factor elF3. Nucleic Acids Res 36: 4708-4718. doi:10.1093/nar/gkn454

Lee AS, Kranzusch PJ, Cate JH. 2015. elF3 targets cell-proliferation messenger RNAs for translational activation or repression. Nature 522: 111-114. doi:10.1038/nature14267

Lennox $A L$, Hoye $M L$, Jiang $R$, Johnson-Kerner $B L$, Suit LA, Venkataramanan S, Sheehan CJ, Alsina FC, Fregeau B, Aldinger KA, et al. 2020. Pathogenic DDX3X mutations impair RNA metabolism and neurogenesis during fetal cortical development. Neuron 106: 404-420. doi:10.1016/j.neuron.2020.01.042

Letunic I, Bork P. 2019. Interactive tree of life (iTOL) v4: recent updates and new developments. Nucleic Acids Res 47: W256-W259. doi:10.1093/nar/gkz239

Lizio M, Harshbarger J, Shimoji H, Severin J, Kasukawa T, Sahin S, Abugessaisa I, Fukuda S, Hori F, Ishikawa-Kato S, et al. 2015. Gateways to the FANTOM5 promoter level mammalian expression atlas. Genome Biol 16: 22. doi:10.1186/s13059-014-0560-6

Love Ml, Huber W, Anders S. 2014. Moderated estimation of fold change and dispersion for RNA-seq data with DESeq2. Genome Biol 15: 550. doi:10.1186/s13059-014-0550-8

Matsumura T, Endo T, Isotani A, Ogawa M, Ikawa M. 2019. An azoospermic factor gene, $D d x 3 y$ and its paralog, $D d x 3 x$ are dispensable in germ cells for male fertility. J Reprod Dev 65: 121-128. doi:10.1262/jrd.2018-145

McGlincy NJ, Ingolia NT. 2017. Transcriptome-wide measurement of translation by ribosome profiling. Methods 126: 112-129. doi:10 .1016/j.ymeth.2017.05.028

Natsume T, Kiyomitsu T, Saga Y, Kanemaki MT. 2016. Rapid protein depletion in human cells by auxin-inducible degron tagging with short homology donors. Cell Rep 15: 210-218. doi:10.1016/j celrep.2016.03.001

Oh S, Flynn RA, Floor SN, Purzner J, Martin L, Do BT, Schubert S, Vaka D, Morrissy S, Li Y, et al. 2016. Medulloblastoma-associated DDX3 variant selectively alters the translational response to stress. Oncotarget 7: 28169-28182. doi:10.18632/oncotarget.8612

Ojha J, Secreto CR, Rabe KG, Van Dyke DL, Kortum KM, Slager SL, Shanafelt TD, Fonseca R, Kay NE, Braggio E. 2015. Identification of recurrent truncated DDX3X mutations in chronic lymphocytic leukaemia. Br J Haematol 169: 445-448. doi:10.1111/bjh.13211

Patmore DM, Jassim A, Nathan E, Gilbertson RJ, Tahan D, Hoffmann N, Tong Y, Smith KS, Kanneganti T-D, Suzuki H, et al. 2020. DDX3X suppresses the susceptibility of hindbrain lineages to medulloblastoma. Dev Cell 54: 455-470. doi:10.1016/j.devcel 2020.05.027

Ramathal C, Angulo B, Sukhwani M, Cui J, Durruthy-Durruthy J, Fang F, Schanes $P$, Turek P, Orwig K, Pera RR. 2015. DDX3Y gene rescue of a $Y$ chromosome AZFa deletion restores germ cell formation and transcriptional programs. Sci Rep 5: 15041. doi:10.1038/srep15041

Rauschendorf MA, Zimmer J, Hanstein R, Dickemann C, Vogt PH. 2011. Complex transcriptional control of the AZFa gene DDX3Y in human testis. Int J Androl 34: 84-96. doi:10.1111/j.1365-2605 .2010.01053.x

Rauschendorf MA, Zimmer J, Ohnmacht C, Vogt PH. 2014. DDX3X, the $X$ homologue of AZFa gene DDX3Y, expresses a complex pattern of transcript variants only in the male germ line. Mol Hum Reprod 20: 1208-1222. doi:10.1093/molehr/gau081

Ruzzo EK, Perez-Cano L, Jung JY, Wang LK, Kashef-Haghighi D, Hartl C, Singh C, Xu J, Hoekstra JN, Leventhal O, et al.
2019. Inherited and de novo genetic risk for autism impacts shared networks. Cell 178: 850-866.e26. doi:10.1016/j.cell.2019 .07 .015

Scala M, Torella A, Severino M, Morana G, Castello R, Accogli A, Verrico A, Vari MS, Cappuccio G, Pinelli M, et al. 2019. Three de novo DDX3X variants associated with distinctive brain developmental abnormalities and brain tumor in intellectually disabled females. Eur J Hum Genet 27: 1254-1259. doi:10.1038/s41431019-0392-7

Sekiguchi T, lida H, Fukumura J, Nishimoto T. 2004. Human DDX3Y, the $Y$-encoded isoform of RNA helicase DDX3, rescues a hamster temperature-sensitive ET24 mutant cell line with a DDX3X mutation. Exp Cell Res 300: 213-222. doi:10.1016/j.yexcr.2004.07.005

Sharma D, Jankowsky E. 2014. The Ded1/DDX3 subfamily of DEADbox RNA helicases. Crit Rev Biochem Mol Biol 49: 343-360. doi:10.3109/10409238.2014.931339

Shih JW, Wang WT, Tsai TY, Kuo CY, Li HK, Wu Lee YH. 2012. Critical roles of RNA helicase DDX3 and its interactions with elF4E/PABP1 in stress granule assembly and stress response. Biochem $J$ 441: 119-129. doi:10.1042/BJ20110739

Snijders Blok L, Madsen E, Juusola J, Gilissen C, Baralle D, Reijnders MR, Venselaar H, Helsmoortel C, Cho MT, Hoischen A, et al. 2015. Mutations in DDX3X are a common cause of unexplained intellectual disability with gender-specific effects on Wnt signaling. Am J Hum Genet 97: 343-352. doi:10.1016/j.ajhg .2015.07.004

Stransky N, Egloff AM, Tward AD, Kostic AD, Cibulskis $K$, Sivachenko A, Kryukov GV, Lawrence MS, Sougnez C, McKenna A, et al. 2011. The mutational landscape of head and neck squamous cell carcinoma. Science 333: 1157-1160. doi:10 $.1126 /$ science. 1208130

Takata A, Miyake N, Tsurusaki Y, Fukai R, Miyatake S, Koshimizu E, Kushima I, Okada T, Morikawa M, Uno Y, et al. 2018. Integrative analyses of de novo mutations provide deeper biological insights into autism spectrum disorder. Cell Rep 22: 734-747. doi:10 .1016/j.celrep.2017.12.074

Torchiano M. 2020. Effsize-a package for efficient effect size computation version 0.8.0. https://github.com/mtorchiano/effsize/ releases/tag/v0.8.0

Uhlen M, Fagerberg L, Hallstrom BM, Lindskog C, Oksvold P, Mardinoglu A, Sivertsson A, Kampf C, Sjostedt E, Asplund A, et al. 2015. Proteomics. Tissue-based map of the human proteome. Science 347: 1260419. doi:10.1126/science.1260419

Vakilian H, Mirzaei M, Sharifi Tabar M, Pooyan P, Habibi Rezaee L, Parker L, Haynes PA, Gourabi H, Baharvand H, Salekdeh GH. 2015. DDX3Y, a male-specific region of $Y$ chromosome gene, may modulate neuronal differentiation. J Proteome Res 14: 3474-3483. doi:10.1021/acs.jproteome.5b00512

Waldman T, Kinzler KW, Vogelstein B. 1995. p21 is necessary for the p53-mediated $\mathrm{G}_{1}$ arrest in human cancer cells. Cancer Res 55: 5187-5190.

Wang T, Birsoy K, Hughes NW, Krupczak KM, Post Y, Wei JJ, Lander ES, Sabatini DM. 2015. Identification and characterization of essential genes in the human genome. Science 350: 10961101. doi:10.1126/science.aac7041

Wang $X$, Posey JE, Rosenfeld JA, Bacino CA, Scaglia F, Immken L, Harris JM, Hickey SE, Mosher TM, Slavotinek A, et al. 2018. Phenotypic expansion in DDX3X - a common cause of intellectual disability in females. Ann Clin Transl Neurol 5: 1277-1285. doi:10 $.1002 / a c n 3.622$

Yuen RC, Merico D, Bookman M, Howe JL, Thiruvahindrapuram B, Patel RV, Whitney J, Deflaux N, Bingham J, Wang Z, et al. 2017. Whole genome sequencing resource identifies 18 new candidate genes for autism spectrum disorder. Nat Neurosci 20: 602-611. doi:10.1038/nn.4524 

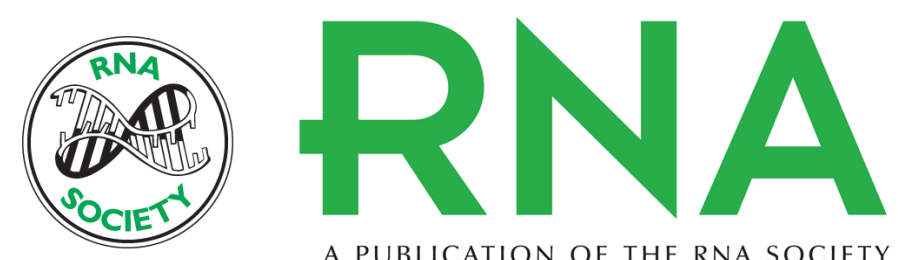

A PUBLICATION OF THE RNA SOCIETY

\section{DDX3X and DDX3Y are redundant in protein synthesis}

Srivats Venkataramanan, Margaret Gadek, Lorenzo Calviello, et al.

RNA 2021 27: 1577-1588 originally published online September 17, 2021

Access the most recent version at doi:10.1261/rna.078926.121

Supplemental Material

References

Open Access

Creative Commons

License

Email Alerting
Service
http://rnajournal.cshlp.org/content/suppl/2021/09/17/rna.078926.121.DC1

This article cites 49 articles, 9 of which can be accessed free at: http://rnajournal.cshlp.org/content/27/12/1577.full.html\#ref-list-1

Freely available online through the RNA Open Access option.

This article, published in RNA, is available under a Creative Commons License (Attribution 4.0 International), as described at http://creativecommons.org/licenses/by/4.0/.

To subscribe to RNA go to:

http://rnajournal.cshlp.org/subscriptions

(C) 2021 Venkataramanan et al.; Published by Cold Spring Harbor Laboratory Press for the RNA Society 